09,12

\title{
Фотонное эхо на локализованных экситонах в полупроводниковых наноструктурах
}

\author{
(C) С.В. Полтавцев ${ }^{1,2}$, И.А. Югова ${ }^{2}$, И.А. Акимов ${ }^{1,3,1}$, Д.Р. Яковлев ${ }^{1,3}$, М. Bayer $r^{1,3}$ \\ ${ }^{1}$ Experimentelle Physik 2, Technische Universität Dortmund D-44227, \\ Dortmund, Germany \\ ${ }^{2}$ Санкт-Петербургский государственный университет, \\ Санкт-Петербург, Петергоф, Россия \\ ${ }^{3}$ Физико-технический институт им. А.Ф. Иофффе РАН, \\ Санкт-Петербург, Россия \\ E-mail: ilja.akimov@tu-dortmund.de
}

\begin{abstract}
Представлен обзор по спектроскопии фотонного эха при резонансном возбуждении экситонных комплексов в полупроводниковых наноструктурах. Использвание импульсной методики четырехволнового смешивания с гетеродинным детектированием позволяет измерять когерентный отклик системы с пикосекундным временным разрешением. Показано, что при резонансном селективном импульсном возбуждении локализованных экситонных комплексов когерентный сигнал определяется фотонным эхом вследствие неоднородного уширения оптических переходов. Для случая резонансного возбуждения трионов или экситонов, связанных на донорах, зеемановское расщепление спиновых уровней резидентных электронов в поперечном магнитном поле приводит к появлению квантовых биений фотонного эха на частоте ларморовой прецессии. При этом в магнитном поле удается осуществить когерентный перенос оптического возбуждения в спиновый ансамбль резидентных электронов и наблюдать долгоживущее фотонное эхо. Данные эксперименты могут быть использованы в качестве спектроскопии с высоким разрешением для измерения расщеплений в основном состоянии системы. В обзоре обсуждаются осцилляции Раби и их затухание при возбуждении мощными оптическими импульсами экситонных комплексов с разной степенью локализации. Показано, что гашение сигнала фотонного эха с увеличением интенсивности возбуждающих импульсов наиболее ярко выражено при возбужденни экситонов, в то время как для трионов и экситонов связанных на донорах, этот эффект значительно слабее.
\end{abstract}

Авторы благодарят за финансовую поддержку Deutsche Forschungsgemeinschaft ICRC TRR-160 (проект A3) и Российский Фонд Фундаментальных Исследований (проект № 15-52-12016 ННИО_а). Работа выполнена при поддержке гранта СПбГУ 11.34.2.2012.

DOI: $10.21883 /$ FTT.2018.08.46254.17Gr

\section{1. Введение}

Когерентная оптическая спектроскопия позволяет получить богатую информацию об энергетической структуре и естественной ширине спектральных линий исследуемой системы. Использование импульсных источников света позволяет проводить эксперименты с временным разрешением и получать информацию о динамических процессах и основных механизмах, приводящих к потере когерентности (фазовой релаксации). Естественным свойством большинства макроскопических систем является неоднородное уширение оптических преходов. В этом случае резонансное возбуждение последовательностью оптических импульсов приводит к появлению фотонного эха - нелинейного когеретного отклика системы в виде запаздывающего светового импульса $[1,2]$. Времена запаздывания, на которых можно наблюдать фотонное эхо, определяются когерентными свойствами локального (одиночного) квантово-механического возбужденного состояния. Таким образом, изучение сигналов фотонного эха позволяет преодолеть неоднородное уширение оптических переходов и получить информацию о когерентной динамике одиночного оптического возбуж- дения в большом ансамбле излучателей. Двухимпульсное и трехимпульсное фотонное эхо активно используют для изучения энергетической структуры и когерентной эволюции оптических возбуждений в атомных системах, редкоземельных кристаллах и полупроводниках [3-5]. Кроме того, фотонное эхо рассматривается в качестве возможного кандидата для реализации оптической памяти в ансамбле излучателей [6].

В полупроводниках элементарными оптическими возбуждениями являются экситонные комплексы (связанные электронно-дырочные пары). Экситоны обладают большой силой осциллятора, что позволяет осуществлять быстрое и эффективное оптическое возбуждение при помощи субпикосекундных импульсов. Следует отметить, что взаимодействие между экситонами в полупроводниковых кристаллах довольно велико, что приводит к сложной динамике оптического когерентного отклика [4]. Более того, такой отклик наблюдается на коротких временах в пределах нескольких пикосекунд. Однако, в случае локализации экситонов многочастичные взаимодействия подавлены, и локализованные экситоны могут рассматриваться как одиночные невзаимодействующие комплексы с дискретным энергетическим спек- 
тром. В простейшем случае резонансного возбуждения экситонный комплекс можно рассматривать как двухуровневую энергетическую систему с основным и возбужденным состояниями. Наличие флуктуаций состава и размера локализующего потенциала приводят к неоднородному уширению оптических переходов, и когерентный оптический отклик представляет собой фотонное эхо $[7,8]$. Ярким примером полупроводниковой системы с локализованными экситонами являются самоорганизованные квантовые точки. Эксперименты по четырехволновому смешению с фемтосекундными импульсами в ансамбле квантовых точек показали, что при низких температурах время когерентности экситона $T_{2}$ может быть сравнимо с его временем жизни $T_{1} \sim 1 \mathrm{~ns}$ [9]. Таким образом, локализация экситонных комплексов приводит к увеличению их времен когерентности. При этом, поскольку многочастичные взаимодействия подавлены, то появляется возможность когерентного управления экситонными состояниями при помощи интенсивных лазерных импульсов. Примером такого когерентного управления может служить демонстрация осцилляций Раби в двухуровневой системе [10,11].

Особый интерес привлекают системы с более чем двумя электронными состояниями, взаимодействующими со светом. Здесь наиболее яркими примерами являются энергетические уровни с порядком $V$ - $(\Lambda-)$ типа, где одно основное (возбужденное) состояние оптически связано с двумя возбужденными (основными) состояниями [12]. Такие схемы уровней позволяют наблюдать различные интересные явления, к которым относятся квантовые биения, когерентное пленение заселенностей и электромагнитно-индуцированная прозрачность $[6,13]$. Основной особенностью $\Lambda$-схемы является длительное время когерентности основных состояний. В полупроводниках $\Lambda$-схема может быть реализована при оптическом возбуждении локализованных резидентных носителей, например, электронов в зоне проводимости или дырок в валентной зоне, с использованием их спиновой степени свободы. В случае резидентных электронов отрицательно заряженный экситон (трион $X^{-}$) и связанный с донором экситон $\left(D^{0} X\right)$ являются возможными оптически возбужденными состояниями [14]. Оптическое управление спина резидентных носителей при помощи коротких пикосекундных импульсов было успешно продемонстрировано в различных полупроводниковых системах [15-17]. Однако большинство исследований четырехволнового смешения и фотонного эха в полупроводниках до сих пор не использовали спиновой степени свободы в основном состоянии [18-21].

В настоящей работе представлен обзор недавних результатов по спектроскопии фотонного эха при резонансном возбуждении локализованных экситонных комплексов в полупроводниках. Особое внимание уделяется системам с резидентными электронами в поперечном магнитном поле. В этом случае последовательный стимулированный рамановский процесс при возбуждении двумя оптическими импульсами позволяет осуществить когерентный перенос оптического возбуждения в спиновый ансамбль резидентных электронов и наблюдать долгоживущее фотонное эхо [22,23]. Долгоживущее эхо отражает локальную динамику основного состояния системы и позволяет получать богатую информацию о спиновых процессах в ансамбле резидентных электронов даже для объектов с большим спектральным уширением оптических переходов [24]. При этом выбор материала и размерность квантовой системы играют важную роль. Это влияет на правила отбора для оптических переходов, степень локализации, а также на процессы спиновой релаксации как для оптически возбужденного, так и для основного состояния. В настоящей статье мы обсудим основные эффекты на примере модельной системы - квантовых ямах $\mathrm{CdTe} /(\mathrm{Cd}, \mathrm{Mg}) \mathrm{Te}$, в которых неоднородное уширение оптических переходов невелико и позволяет селективно возбуждать различные экситонные комплексы с разной степенью локализации [24,25]. При этом мы обсудим также сигналы фотонного эха в гексагональных эпитаксиальных слоях $\mathrm{ZnO}$ [26], квантовых ямах $\mathrm{ZnSe} /(\mathrm{Zn}, \mathrm{Mg})(\mathrm{S}, \mathrm{Se})$ и самоорганизованных квантовых точках (In, Ga)As/GaAs, помещенных в планарные микрорезонаторы для усиления взаимодействия экситонов со светом $[27,28]$. Экспериментальные результаты были получены с использованием четырехволнового смешения с гетеродинным детектированием, которое описано в разд. 2. В разд. 3 рассматриваются долгоживущие сигналы фотонного эха, полученные от ансамбля резидентных электронов. В разд. 4 обсуждаются осцилляции Раби при интенсивном оптическом возбуждении, что является одним из необходимых условий для эффективного оптического контроля квантовых состояний.

\section{2. Техника эксперимента}

Для исследования сигналов четырехволнового смешения и фотонного эха была разработана экспериментальная установка, обладающая высокой чувствительностью и пикосекундным временным разрешением. Оптическая схема установки изображена на рис. 1. Источником пикосекундных лазерных импульсов служит перестраиваемый в диапазоне 700-1000 nm титан-сапфировый лазер Mira-900, накачиваемый излучением твердотельного лазера Verdi-V10 с длиной волны $532 \mathrm{~nm}$. Лазерные импульсы длительностью около 2 ps делятся неполяризующими делителями света на несколько импульсов, включая первый, второй и третий возбуждающие импульсы, а также опорный импульс (Reference). Bce импульсы, кроме первого, задерживаются с помощью оптических линий задержки так, как показано на вставке в рис. 1: второй импульс задерживается на время $\tau_{12}$ относительно первого, третий - на $\tau_{23}$ относительно второго, и опорный - на $\tau_{\text {Ref }}$ относительно первого. Три возбуждающих импульса фокусируются на образце, помещенном в гелиевый заливной криостат и охлажден- 


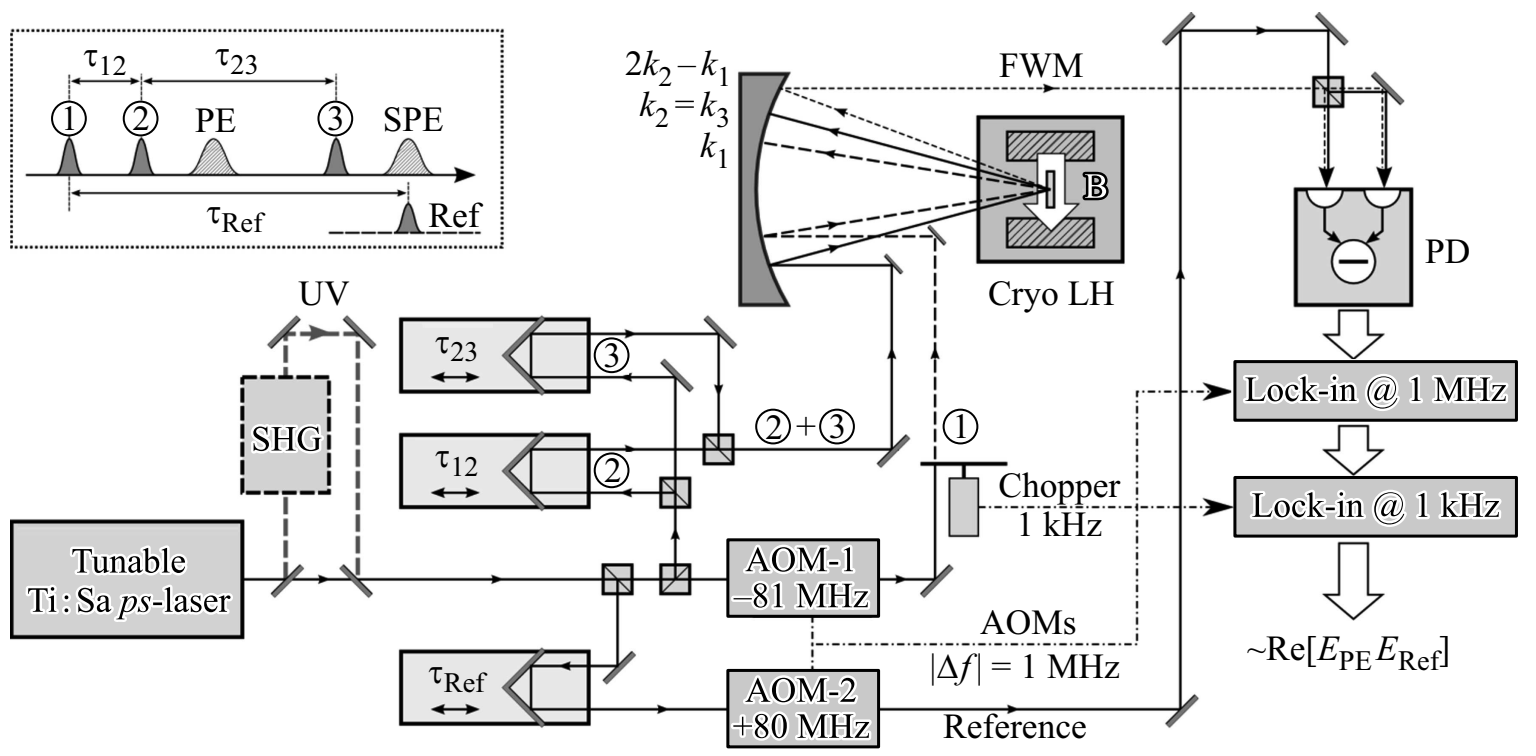

Рис. 1. Оптическая схема установки для измерения фотонного эха с пикосекундным временным разрешением. Обозначения: SHG - генератор второй гармоники, Сryo LH - гелиевый заливной криостат, PD - фотодетектор, AOM - акусто-оптический модулятор, UV - ультрафиолетовое излучение. Вставка: временная диаграмма задержки возбуждающих лазерных импульсов и сигналов эха. Двухимпульсное (PE) и трехимпульсное (SPE) эхо задержаны на время $\tau_{12}$ после второго и третьего импульса соответственно.

ном до температуры около $2 \mathrm{~K}$, в пятно диаметром около $300 \mu \mathrm{m} \mathrm{c}$ помощью сферического зеркала с фокусным расстоянием $500 \mathrm{~mm}$. Все импульсы линейно кополяризованы. Первый и второй импульсы с волновыми векторами $\mathbf{k}_{1}$ и $\mathbf{k}_{2}$ падают на образец под углами соответственно $3^{\circ}$ и $4^{\circ}$. Третий импульс распространяется в том же направлении, что и второй $\left(\mathbf{k}_{3}=\mathbf{k}_{2}\right)$. Сигнал четырехволнового смешения (FWM) собирается в геометрии отражения в направлении $2 \mathbf{k}_{2}-\mathbf{k}_{1}$ с помощью того же сферического зеркала и заводится на два канала балансного фотодетектора с использованием неполяризующего делителя света, делящего интенсивность сигнала в соотношении $1: 1$. На этот же фотодетектор в обход образца заводится и опорный импульс, и измеряется кросс-корреляция опорного импульса с полем четырехволнового смешения.

Для детектирования слабых сигналов четырехволнового смешения используется оптическое гетеродинирование. Оно реализовано с помощью двух акустооптических модуляторов АОМ-1 и АОМ-2, сдвигающих оптические частоты света первого возбуждающего и опорного импульсов на $-81 \mathrm{MHz}$ и $+80 \mathrm{MHz}$ соответственно. Оптическая частота света четырехволнового смешения $v_{\mathrm{GWM}}$ при этом равна $2 v_{2}-v_{1}$, где $v_{1}=v_{0}-81 \mathrm{MHz}, v_{2}=v_{0}\left(v_{0}-\right.$ исходная частота света), что дает $v_{\mathrm{FWM}}=v_{0}+81 \mathrm{MHz}$. В результате интерференции с опорным излучением $\left(v_{\mathrm{Ref}}=v_{0}+80 \mathrm{MHz}\right)$ на фотодетекторе регистрируются оптические биения, в частности, на разностной частоте опорного света и четырехволнового смешения $\Delta f=1 \mathrm{MHz}$. Причем постоянные составляющие, пропорциональные суммар- ной интенсивности света лучей в каждом канале фотодетектора, взаимовычитаются в балансной схеме, а амплитуда оптических биений, наоборот, удваивается. Детектируемая „быстрым“ синхронным детектором на частоте $1 \mathrm{MHz}$, амплитуда оптических биений пропорциональна модулю произведения амплитуд опорного света $E_{\mathrm{Ref}}$ и сигнала четырехволнового смешения $E_{\mathrm{PE}}$ : $\delta I_{\text {Det }} \sim\left|E_{\mathrm{PE}} E_{\mathrm{Ref}}^{*}\right|$. Для уменьшения паразитных помех применяется модуляция интенсивности первого возбуждающего луча с помощью механического прерывателя (чоппера) на частоте около $1 \mathrm{kHz}$. На этой частоте осуществляется вторичное детектирование сигнала с помощью „медленного“ синхронного детектора.

К образцу прикладывается внешнее магнитное поле свехпроводящего магнита, направленное вдоль плоскости структуры величиной до 6 Т. В экспериментах с широкозонными полупроводниковыми структурами $(\mathrm{ZnO}$, $\mathrm{ZnSe}$ ) дополнительно используется генератор второй гармоники (SHG), позволяющий работать с пикосекундными импульсами ультрафиолетового излучения с длиной волны 350-495 nm и длительностью $1.3 \mathrm{~ns}$. При исследовании структур на основе $\mathrm{ZnO}$ с полированной сапфировой подложкой сигнал FWM детектируется в геометрии на пропускание с использованием другого сферического зеркала с фокусным расстоянием $500 \mathrm{~mm}$ и той же схемы детектирования. Объектами наших исследований являются различные эпитаксиальные полупроводниковые гетероструктуры $A_{2} B_{6}$ и $A_{3} B_{5}$, включая квантовые ямы $\mathrm{CdTe} /(\mathrm{Cd}, \mathrm{Mg}) \mathrm{Te}[22-24], \mathrm{ZnSe} /(\mathrm{Zn}, \mathrm{Mg})(\mathrm{S}, \mathrm{Se})$, квантовые точки (In, Ga)As/GaAs [27,28], а также эпитаксиальные слои $\mathrm{ZnO}$ [26]. В квантовых ямах и объем- 


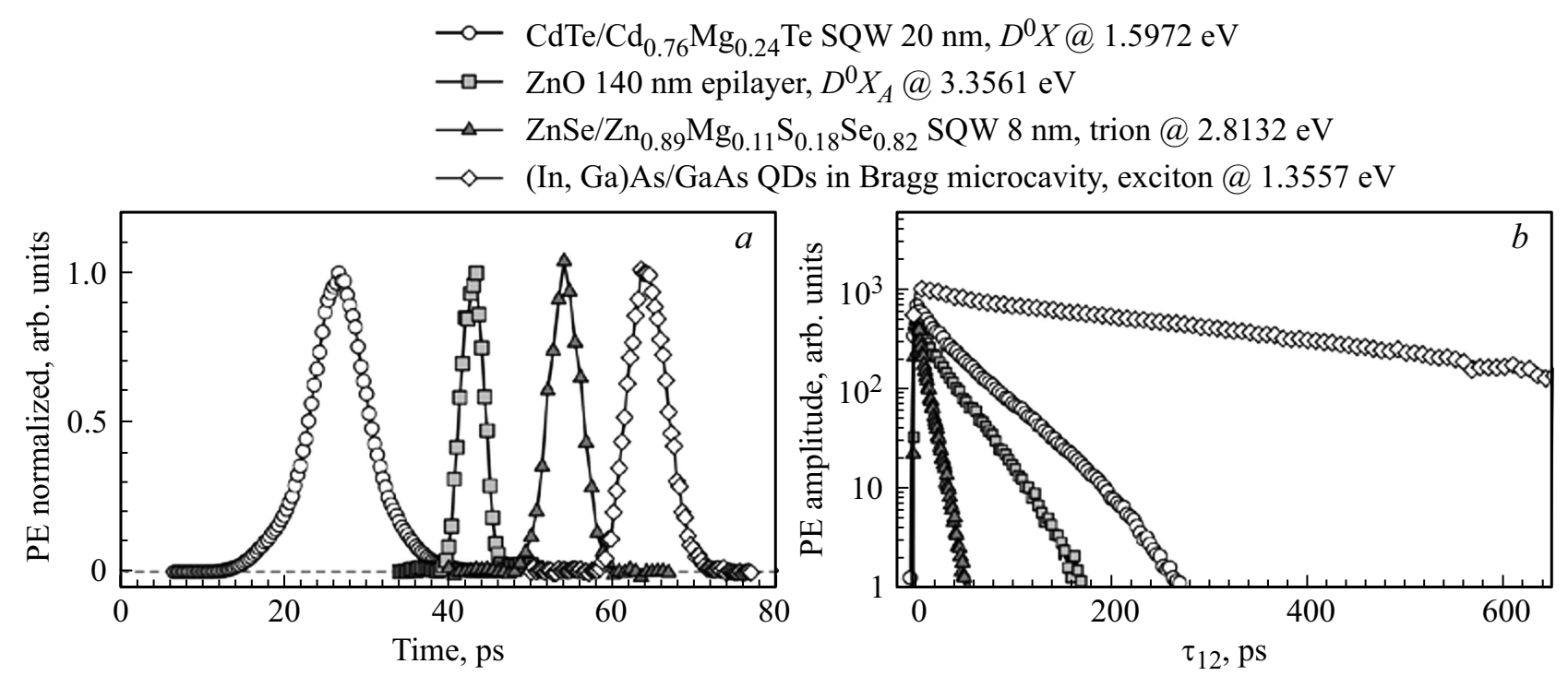

Рис. 2. Измерение временного профиля $(a)$ и затухания амплитуды двухимпульсного фотонного эха $(b)$ на $D^{0} X$ в одиночной квантовой яме $\mathrm{CdTe} /(\mathrm{Cd}, \mathrm{Mg}) \mathrm{Te}$ (кружки), на $D^{0} X$ в эпитаксиальном слое $\mathrm{ZnO}$ (квадраты), на трионе в квантовой яме $\mathrm{ZnSe} /(\mathrm{Zn}, \dot{\mathrm{Mg}})(\mathrm{S}, \dot{\mathrm{Se}})$ (треугольники) и на экситоне в квантовых точках (In, Ga) As/GaAs, помещенных в брегговский микрорезонатор (ромбы).

ных материалах проблем с детектированием сигнала FWM не возникает. Однако в квантовых точках сильное неоднородное уширение существенно уменьшает число квантовых точек, у которых энергия оптического перехода соответствует резонансному возбуждению. Для усиления сигнала FWM квантовые точки выращивают в микрорезонаторах, образованных, например, двумя брегговскими зеркалами, формируемыми в процессе роста структуры. При этом фотонная мода микрорезонатора пропускает пикосекундный лазерный импульс без существенных искажений, так как используемый резонатор обладает малым $Q$-фактором $(Q \approx 100-200)$ и эффектов сильной связи не возникает.

Описанная установка позволяет измерять различные вклады сигнала четырехволнового смешения, в частности, двухимпульсное (PE) и трехимпульсное (SPE) фотонное эхо, распространяющиеся во времени в соответствии со схемой на вставке на рис. 1. Изменение задержки опорного импульса $\tau_{\operatorname{Ref}}$ позволяет измерять временной профиль фотонного эха с пикосекундным разрешением. На рис. 2, а показаны временные профили двухимпульсного фотонного эха, полученные на четырех разных системах: связанном на доноре экситоне $\left(D^{0} X\right)$ в одиночной квантовой яме $\mathrm{CdTe} /(\mathrm{Cd}, \mathrm{Mg}) \mathrm{Te}, D^{0} X$ в эпитаксиальном слое $\mathrm{ZnO}$ толщиной $140 \mathrm{~nm}$, на трионе в квантовой яме $\mathrm{ZnSe} /(\mathrm{Zn}, \mathrm{Mg})(\mathrm{S}, \mathrm{Se})$, а также на экситонах в квантовых точках (In, Ga)As/GaAs, помещенных в брегговский микрорезонатор. Так как измеряется кросскорреляция сигнала FWM с опорным импульсом, то профиль фотонного эха есть результат свертки импульса эха с опорным импульсом. Ширина профиля эха частично отражает неоднородное уширение возбужденного ансамбля, связанное со временем обратимой фазовой релаксации, $\Gamma_{2}^{*} \propto 1 / T_{2}^{*}$. Когда ширина ансамбля значительно уже спектра импульса, профиль эха становится широким. К этому случаю относится эхо на $D^{0} X$ в квантовой яме $\mathrm{CdTe} /(\mathrm{Cd}, \mathrm{Mg}) \mathrm{Te}$, длительность которого составляет 8 ps. Когда же неоднородная ширина спектра сравнима или существенно больше ширины спектра импульса, импульс эха примерно соответствует функции автокорреляции лазерного импульса. Это наблюдается в случае эха от остальных систем на рис. 2, $a$. При этом нужно иметь в виду, что для ультрафиолетового диапазона длительность импульса сокращается в $\sqrt{2}$ раз по сравнению с исходными инфракрасными импульсами. Профиль фотонного эха, однако, может принимать и более сложную форму, когда интенсивность возбуждения значительно увеличивается. Тогда измерение сигнала четырехволнового смешения выходит из режима $\chi^{(3)}$ и переходит в режим осцилляций Раби. Экспериментам такого рода с фотонным эхом посвящен разд. 4 этой работы.

Если изменить одновременно задержку между первым и вторым возбуждающими импульсами и задержку опорного импульса с условием $\tau_{\text {Ref }}=2 \tau_{12}$, то можно измерить затухание амплитуды фотонного эха. На рис. 2, $b$ показана кинетика затухания эха для исследованных четырех систем. Видно, что полученные зависимости могут быть аппроксимированы с помощью экспоненциального затухания $\sim \exp \left(-2 \tau_{12} / T_{2}\right), \quad$ из которого извлекается время необратимой фазовой релаксации $T_{2}$ изучаемого резонанса. Оно соответствует однородной ширине спектральной линии резонанса $\Gamma_{2}=2 \hbar / T_{2}$ (полная ширина на уровне половинной амплитуды). Самое большое время $T_{2}=750$ ps наблюдается на ансамбле квантовых точек (In, Ga)As/GaAs и соответствует $\Gamma_{2}=1.8 \mu \mathrm{eV}$. Самое короткое время, 

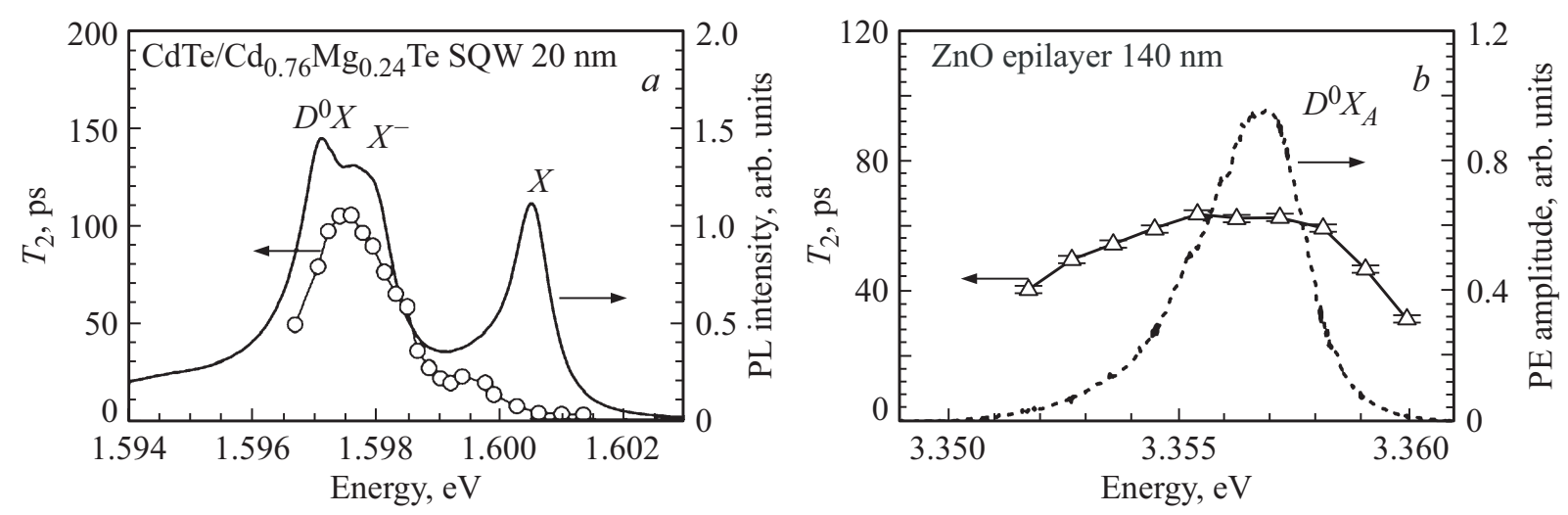

Рис. 3. Спектральная зависимость времени необратимой фазовой релаксации $T_{2}$, измеренные в одиночной квантовой яме $\mathrm{CdTe} /(\mathrm{Cd}, \mathrm{Mg}) \mathrm{Te}(a)$ и в эпитаксиальном слое $\mathrm{ZnO}$ толщиной $140 \mathrm{~nm}(b)$. Сплошная линия на панели $(a)$ соответствует спектру фотолюминесценции, в котором видны: экситон $(X)$, трион $\left(X^{-}\right)$и связанный на доноре экситон $\left(D^{0} X\right)$. Пунктирная линия на панели $(b)$ - это спектр фотонного эха, измеренный при задержке $\tau_{12}=27 \mathrm{ps}$. Символами показаны значения $T_{2}$.

в данном случае, $T_{2}=16$ ss наблюдается для трионов в квантовой яме $\mathrm{ZnSe} /(\mathrm{Zn}, \mathrm{Mg})(\mathrm{S}, \mathrm{Se})$, для которых $\Gamma_{2}=82 \mu \mathrm{eV}$. Время обратимой фазовой релаксации $T_{2}^{*}$ и время необратимой фазовой релаксации $T_{2}$ в общем случае не коррелируют, и могут быть независимо измерены с помощью фотонного эха.

С помощью перестройки длины волны возбуждающих импульсов можно измерить спектральную зависимость кинетики затухания фотонного эха. На рис. 3 изображены полученные таким образом спектральные зависимости $T_{2}$ для одиночной квантовой ямы $\mathrm{CdTe} /(\mathrm{Cd}, \mathrm{Mg}) \mathrm{Te}$ и эпитаксиального слоя $\mathrm{ZnO}$. Интересным фактом является немонотонность обеих зависимостей в окрестности резонанса $D_{0} X$, где время $T_{2}$ в области низких энергий сокращается. Это противоречит простой гипотезе об увеличении времени когерентности для экситонных комплексов с меньшей энергией оптического перехода изза большей локализации, которая, как правило, хорошо работает. К связанным на донорах экситонам в данных случаях она, по-видимому, не применима.

\section{3. Долгоживущее эхо от ансамбля резидентных электронов}

Исследования, описанные в этом разделе, выполнялись в режиме слабого возбуждения. Это соответствует режиму $\chi^{(3)}$, когда интенсивность сигнала четырехволнового смешения зависит линейно от интенсивности каждого из импульсов (энергия импульса $\sim 10-100 \mathrm{~nJ} \cdot \mathrm{cm}^{-2}$ ).

Рассмотрим фотонное эхо от локализованных трионов на примере квантовой ямы $\mathrm{CdTe} /(\mathrm{Cd}, \mathrm{Mg}) \mathrm{Te}$. Структура энергетических уровней и оптические переходы показаны на рис. 4, $a$. Основное состояние соответствует резидентному электрону и определяется дублетом с электронным спином $S=1 / 2$. Оптически возбужденное состояние с наименьшей энергией соответствует локализованному триону с нулевым электронным спином (синглетное состояние). Таким образом, угловой момент триона $J=3 / 2$ задается тяжелой дыркой и
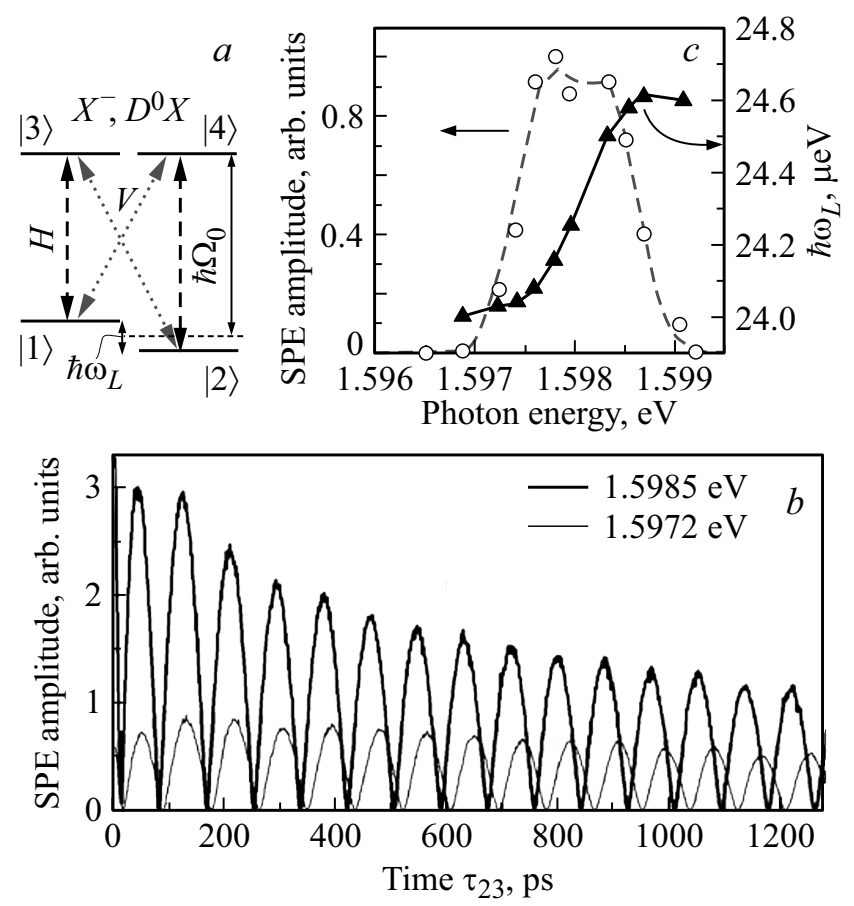

Рис. 4. $a-$ схема энергетических уровней и оптических переходов при возбуждении локализованного триона $\left(X^{-}\right)$или экситона, связанного на доноре $\left(D^{0} X\right)$, в синглетном состоянии в квантовой яме $\mathrm{CdTe} /(\mathrm{Cd}, \mathrm{Mg}) \mathrm{Te}$. $H$ и $V$ соответствуют линейной поляризации оптических переходов параллельно и перпендикулярно магнитному полю, соответственно. $b-$ зависимость амплитуды SPE эха от времени $\tau_{23}$ при резонансном возбуждении комплексов $X^{-}(1.5985 \mathrm{eV})$ и $D^{0} X(1.5972 \mathrm{eV})$. $T=2 \mathrm{~K}, B=260 \mathrm{mT}, \tau_{12}=27 \mathrm{ps}$. Последовательность импульсов имеет поляризационную схему $H V V . c-$ спектральная зависимость амплитуды долгоживущего фотонного эха SPE (кружки) и зеемановского расщепления в основном состоянии $\hbar \omega_{L}$ (треугольники). 
возбужденное состояние тоже представляет собою дублет. В поперечном магнитном поле $\mathbf{B}$, приложенном в плоскости квантвой ямы (перпендикулярно направлению роста структуры), электронные спиновые состояния расщепляются на энергию $\hbar \omega_{L}=g \mu_{\mathrm{B}} B$, где $\omega_{L}$ - частота ларморовой прецессии, $g$ - электронный $g$-фактор, $\mu_{\mathrm{B}}$ - магнетон Бора. Расщепление трионного дублета невелико из-за сильной анизотропии $g$-фактора у тяжелых дырок в структурах с квантовыми ямами [29]. Резонансное возбуждение трионов происходит при помощи световых импульсов, распространяющихся вдоль направления роста структуры, т. е. перпендикулярно направлению магнитного поля (геометрия Фойхта). В такой геометрии оптические переходы допускаются между всеми четырьмя состояниями, которые определяются проекцией углового момента на направление магнитного поля. Можно считать, что оптические переходы линейно поляризованы вдоль $(H)$ или перпендикулярно $(V)$ направлению магнитного поля, как это показано на рис. 4, $a$. Аналогичная схема уровней и правила отбора для оптических переходов реализуется для случая связанных на донорах экситонов. Поэтому наше рассмотрение относится не только к трионам, но и к связанным на донорах экситонам. Более того, аналогичная схема энергетических уровней в поперечном магнитном поле и правила отбора для оптических переходов реализуется для комплекса $D^{0} X$ в гексагональном объемном кристалле $\mathrm{ZnO}$ в случае, если оптическое возбуждение направлено вдоль оси $c$ кристалла. В данном случае кристаллическое поле приводит к расщеплению дырочных состояний в валентной зоне и играет ту же самую роль, что и квантующий потенциал в квантовой яме.

Для демонстрации долгоживущего эха мы осуществляем возбуждение системы при помощи последовательности из трех лазерных импульсов. Правильный выбор поляризации света в последовательности импульсов обеспечивает дополнительную селективность между различными путями возбуждения [24]. Мы рассмотрим поляризационную последовательность HVV (импульс 1 поляризован в направлении $H$, а импульсы 2 и $3-$ в направлении $V$ ). Это соответствует наиболее интересному случаю, когда задействованы все переходы, а когерентная суперпозиция одной пары состояний переносится на другую пару после каждого события возбуждения в ступенчатом режиме.

В данном случае, первый импульс $(H)$ приводит к возбуждению трионов при помощи оптических переходов между состояниями $|1\rangle$ и $|3\rangle$ на частоте $\Omega_{0}-\omega_{L} / 2$ или $|2\rangle$ и $|4\rangle$ на частоте $\Omega_{0}+\omega_{L} / 2$. Здесь $\Omega_{0}$ соответствует резонансной частоте триона в отсутствие магнитного поля $\left(\omega_{L}=0\right)$. Первый импульс создает когерентные суперпозиции между парами состояний $|1\rangle-|3\rangle$ и $|2\rangle-|4\rangle$, т. е. оптическую поляризацию. На языке матрицы плотности эта поляризация соответствует недиагональным элементам $\rho_{13}$ и $\rho_{24}$. При этом предполагается, что до прихода первого импульса система находится в основном состоянии с нулевой спиновой поляризацией, т.е. зеемановское расщепление уровней $\hbar \omega_{L}$ мало по сравнению с тепловой энергией $k_{\mathrm{B}} T$, и единственные ненулевые элементы матрицы плотности $\rho_{11}=\rho_{22}=1 / 2$. Здесь $k_{\mathrm{B}}$ - постоянная Больцмана, а $T-$ температура кристалла. Это условие хорошо выполняется в небольших магнитных полях до $1 \mathrm{~T}$ при $T=2 \mathrm{~K}$.

Будем считать, что когерентность каждого возбужденного триона сохраняется к приходу второго оптического импульса несмотря на то, что макроскопическая поляризация среды быстро исчезает из-за наличия неоднородного уширения оптических переходов (обратимая дефазировка). Второй импульс ( $V$-поляризация) стимулирует оптический переход вниз, в основное состояние системы, таким образом, что оптические когерентности $\rho_{13}$ и $\rho_{24}$ трансформируются в спиновую когерентность в электронном ансамбле $\rho_{12}$. В этом состоянии процесс оптической дефазировки замораживается, и дальнейшая эволюция системы определяется лишь спиновой динамикой локализованных электронов во внешнем магнитном поле. При этом интересно отметить, что время спиновой релаксации электронов может превосходить время жизни трионов на несколько порядков [30]. Третий импульс с $V$-поляризацией снова возбуждает трионы, создавая тем самым оптическую поляризацию $\rho_{42}$ и $\rho_{31}$. Это запускает процесс рефазировки и приводит к наблюдению долгоживущего фотонного эха.

Для случая, когда зеемановское расщепление электронных уровней в основном состоянии меньше спектральной ширины возбуждающего лазера $(\sim 1 \mathrm{meV})$ и неоднородного уширения оптических переходов, сигнал трехимпульсного фотонного эха хорошо описывается импульсами гауссовой формы с амплитудой

$$
P \propto e^{-\frac{2 \tau_{12}}{T_{2}}}\left[e^{-\frac{\tau_{23}}{\tau_{T}}} \cos \left(\omega_{L} \tau_{12}\right)+e^{-\frac{\tau_{23}}{T_{2 e}}} \cos \left(\omega_{L}\left(\tau_{12}+\tau_{23}\right)\right)\right]
$$

где $\tau_{T}$ - время жизни трионного спина. Выражение (1) получается путем решения уравнений Линблада в приближении коротких прямоугольных импульсов [23,24], длительность которых мала по сравнению с периодом ларморовой прецессии спина в основном состоянии $T_{L}=2 \pi / \omega_{L}$. В свою очередь, считается, что $T_{L}$ короче, чем время между первыми двумя импульсами $\tau_{12}$. Выражение (1) содержит два вклада. Первое слагаемое с правой стороны отвечает за спиновую релаксацию и рекомбинацию трионов, которая происходит быстро (до 100 ps в квантовых ямах $\mathrm{CdTe} /(\mathrm{Cd}, \mathrm{Mg}) \mathrm{Te}$ ). Второе слагаемое с правой стороны определяется временем поперечной спиновой релаксации в ансамбле локализованных резидентных электронов $T_{2 e}$. Именно это слагаемое отвечает за долгоживущий сигнал фотонного эха, который наблюдается в случае если $T_{2 e} \gg \tau_{T}$.

Из выражения (1) следует, что амплитуда трехимпульсного эха в магнитном поле осциллирует на частоте ларморовой прецессии. Таким образом, имеется возмож- 

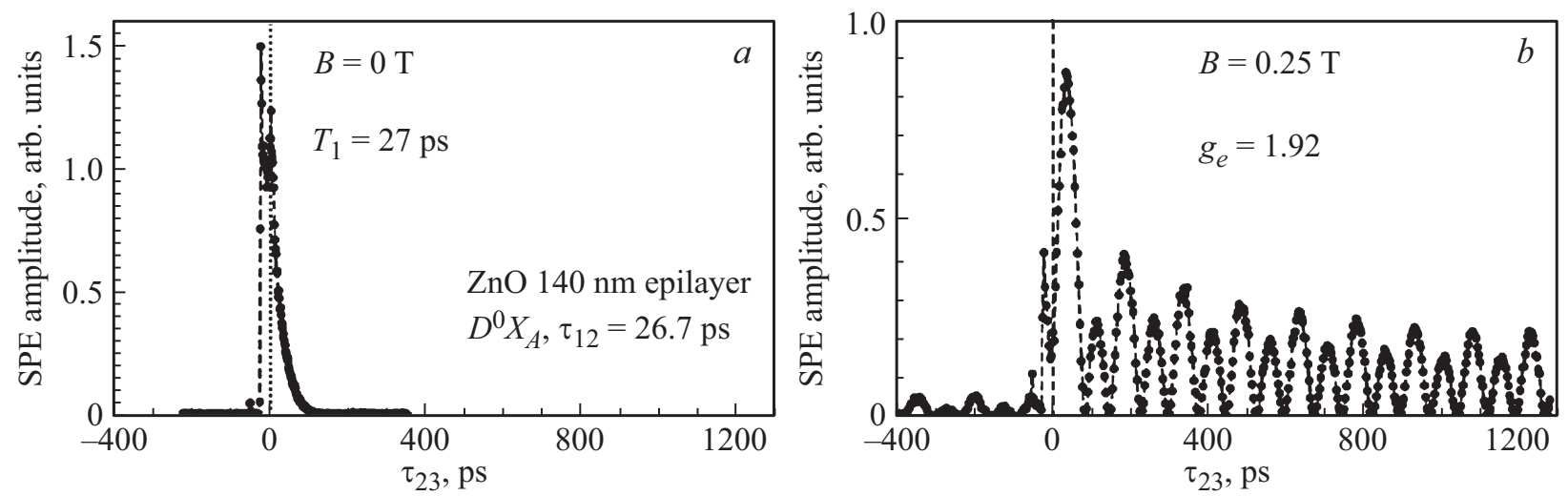

Рис. 5. Зависимость пика SPE от времени $\tau_{23}$ при резонансном возбуждении комплекса $D^{0} X_{A}$ (энергия фотона $3.3566 \mathrm{eV}$ ) в эпитаксиальном слое $\mathrm{ZnO}$ толщиной $140 \mathrm{~nm} . T=2 \mathrm{~K}, \tau_{12}=27 \mathrm{ps}$. Последовательность импульсов имеет поляризацию $H V V$. $B=0(a), B=250 \mathrm{mT}(b)$.

ность измерить величину зеемановского расщепления в основном состоянии при селективном резонансном возбуждении оптическими импульсами. Следует отметить, что измерение возможно даже в том случае, если величина однородного уширения $2 \hbar / T_{2}$ превосходит величину энергетического расщепления, поскольку долгоживущий сигнал определяется исключительно уширением уровней в основном состоянии. Таким образом, данная методика может использоваться в качестве когерентной оптической спектроскопии с высоким спектральным разрешением.

Динамика долгоживущего фотонного эха при резонансном возбуждении трионов и экситонов, связанных на донорах, в квантовой яме $\mathrm{CdTe} /(\mathrm{Cd}, \mathrm{Mg}) \mathrm{Te}$ представлена на рис. 4, b. При этом варьировалось время задержки между вторым и третьим оптическими импульсами и измерялась величина сигнала в максимуме пика фотонного эха (опорный импульс следовал за положением фотонного эха $\left.\tau_{\mathrm{Ref}}=2 \tau_{12}+\tau_{23}\right)$. Величина внешнего магнитного поля составляла $B=260 \mathrm{mT}$, а задержка между первым и вторым импульсом - $\tau_{12}=27$ ps. Из рис. $4, b$ следует, что при изменении задержки $\tau_{23}$, наблюдается осциллирующий сигнал, который затухает на временах порядка нескольких наносекунд, что существенно длиннее времени оптической когерентности $T_{2}$ в этом же образце, показанного для разных энергий возбуждения на рис. 3,a. Отметим, что долгоживущий сигнал наблюдается только в присутствии магнитного поля при оптическом возбуждении в спектральном диапазоне $1.597-1.599 \mathrm{eV}$, т. е. при резонансном воздействии на комплексы $X^{-}$и $D^{0} X$. Очевидно, что для получения долгоживущего сигнала необходимо использовать оптические переходы с резидентными электронами в основном состоянии.

Используя уравнение (1), мы получили спектральную зависимость долгоживущей амплитуды SPE, частоты колебаний $\omega_{L}$, а также времени затухания $T_{2 e}$. Спектральная зависимость для зеемановского расщепления при $B=260$ mТ представлена на рис. 4,c. Наиболее яркой особенностью является изменение частоты колебаний с энергией фотонов, демонстрирующее ступенчатое поведение, т.е. $\hbar \omega_{L}$ увеличивается с 24.0 до $24.6 \mu \mathrm{eV}$, когда энергия возбуждения перемещается от $D^{0} X$ резонанса к $X^{-}$. По этим данным мы оценили $g$-фактор: $|g|=1.595$ и 1.635 для резидентных электронов, связанных с донором и локализованных на флуктуациях потенциала, соответственно. Таким образом, для случая квантовой ямы $\mathrm{CdTe} /(\mathrm{Cd}, \mathrm{Mg}) \mathrm{Te}$ мы измерили расщепление между зеемановскими подуровнями электронов в основном состоянии с разрешением выше $1 \mu \mathrm{eV}$. Это позволило найти различия между двумя подансамблями электронов: электронами, связанными на донорах, и электронами, локализованными на флуктуациях потенциала квантовой ямы.

Отметим, что трехимпульсное долгоживущее эхо, чья динамика определяется спиновой когерентностью в основном состоянии, наблюдается также и в других полупроводниковых системах. Одним из ярких примеров является объемный $\mathrm{ZnO}$ при резонансном возбуждении $A$-экситонов, связанных на донорах (комплекс $D^{0} X_{A}$ ). Результаты измерения в эпитаксиальном слое толщиной $140 \mathrm{~nm}$ показаны на рис. 5. При этом гексагональная ось кристалла (ось $c$ ) направлена перпендикулярно плоскости образца и, соответственно, практически совпадает с направлением распространения возбуждающих импульсов. В отсутствие магнитного поля сигнал $\mathrm{SPE}$ затухает экспоненциально при увеличении задержки $\tau_{23}$ со временем жизни комплекса $D^{0} X_{A}$, т. е. анализа $\mathrm{SPE} \sim \exp \left(-\tau_{23} / T_{1}\right)$. Время затухания соответствует $T_{1}=27 \mathrm{ps}$ [26]. Приложение магнитного поля приводит к появлению долгоживущего осциллирующего сигнала. Из периода осцилляций $T_{L}=150 \mathrm{ps}$ при $B=250 \mathrm{mT}$ мы определяем $|g|=1.92$, который соответствует электронному $g$-фактору. Заметим, что $T_{2 e}$ в $\mathrm{ZnO}$ превышает это время в квантовой яме $\mathrm{CdTe} /(\mathrm{Cd}, \mathrm{Mg}) \mathrm{Te}$, что, повидимому, обусловлено меньшим разбросом $g$-факторов в ансамбле электронов, связанных на донорах. Как видно из рис. 5, $b$ осциллирующий сигнал наблюдается даже 

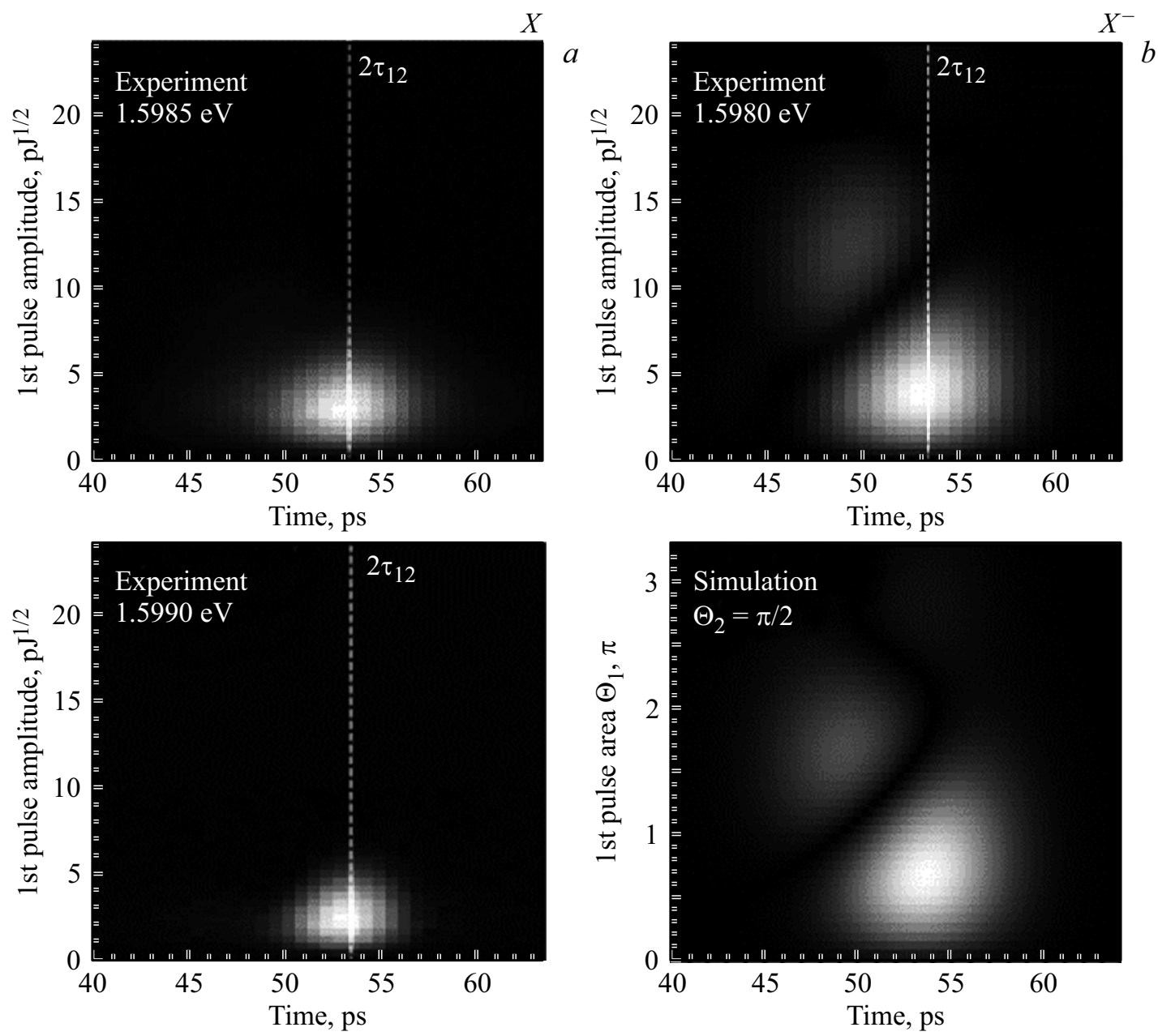

Рис. 6. Осцилляции Раби в амплитуде фотонного эха, измеренные на одиночной квантовой яме $\mathrm{CdTe} /(\mathrm{Cd}, \mathrm{Mg}) \mathrm{Te}: a-$ вариация амплитуды первого возбуждающего импульса на локализованном экситоне в двух спектральных точках; $b, c-$ вариация амплитуды первого и второго импульсов, соответственно, на трионе; $d-$ вариация амплитуды первого импульса на $D^{0} X$. Верхние панели $(b-d)$ - экспериментальные данные, нижние панели - теоретическое моделирование. Площади импульсов измеряются в единицах $\pi$.

при отрицательных задержках. Этот диапазон задержек соответствует большим значениям $\tau_{23}$, которые сравнимы с периодом повторения лазерных импульсов (13 ns). В этом случае возможна реализация интересного режима, схожего с эффектом резонансного спинового усиления [31], в котором накопление спиновой поляризации от последовательности импульсов должно приводить к усилению или ослаблению сигналов фотонного эха в зависмости от величины магнитного поля.

\section{4. Осцилляции Раби}

При увеличении интенсивности возбуждения изучаемого оптического перехода возможен переход из режима измерения фотонного эха $\chi^{(3)}$ в режим когерентных осцилляций Раби, которые можно наблюдать в некоторых исследуемых системах $[25,27,28]$. Поскольку для генерации фотонного эха требуется как минимум два возбуждающих импульса, то варьироваться может амплитуда каждого из них. В оптической схеме для этого используются аттенюаторы в каждом из каналов возбуждения, которые управляются автоматически. Поэтому эксперимент состоит в последовательном увеличении амплитуды одного из возбуждающих импульсов и измерении временного профиля фотонного эха, что приводит к получению двумерного изображения осцилляций Раби в амплитуде эха. Измерения проводились в нулевом магнитном поле, что позволяет упростить энергетическую схему оптических переходов до двухуровневой системы с одиночными основным и возбужденным состояниями.

На рис. 6. показаны экспериментальные данные и теоретическое моделирование этих данных, полученных на различных оптических переходах в одиночной квантовой яме $\mathrm{CdTe} /(\mathrm{Cd}, \mathrm{Mg}) \mathrm{Te}$ [25]. Как видно из этих измерений, картина осцилляций Раби очень чувствительна к энергии возбуждения, которую можно сопоставить со спектром фотолюминесценции, показанным на 

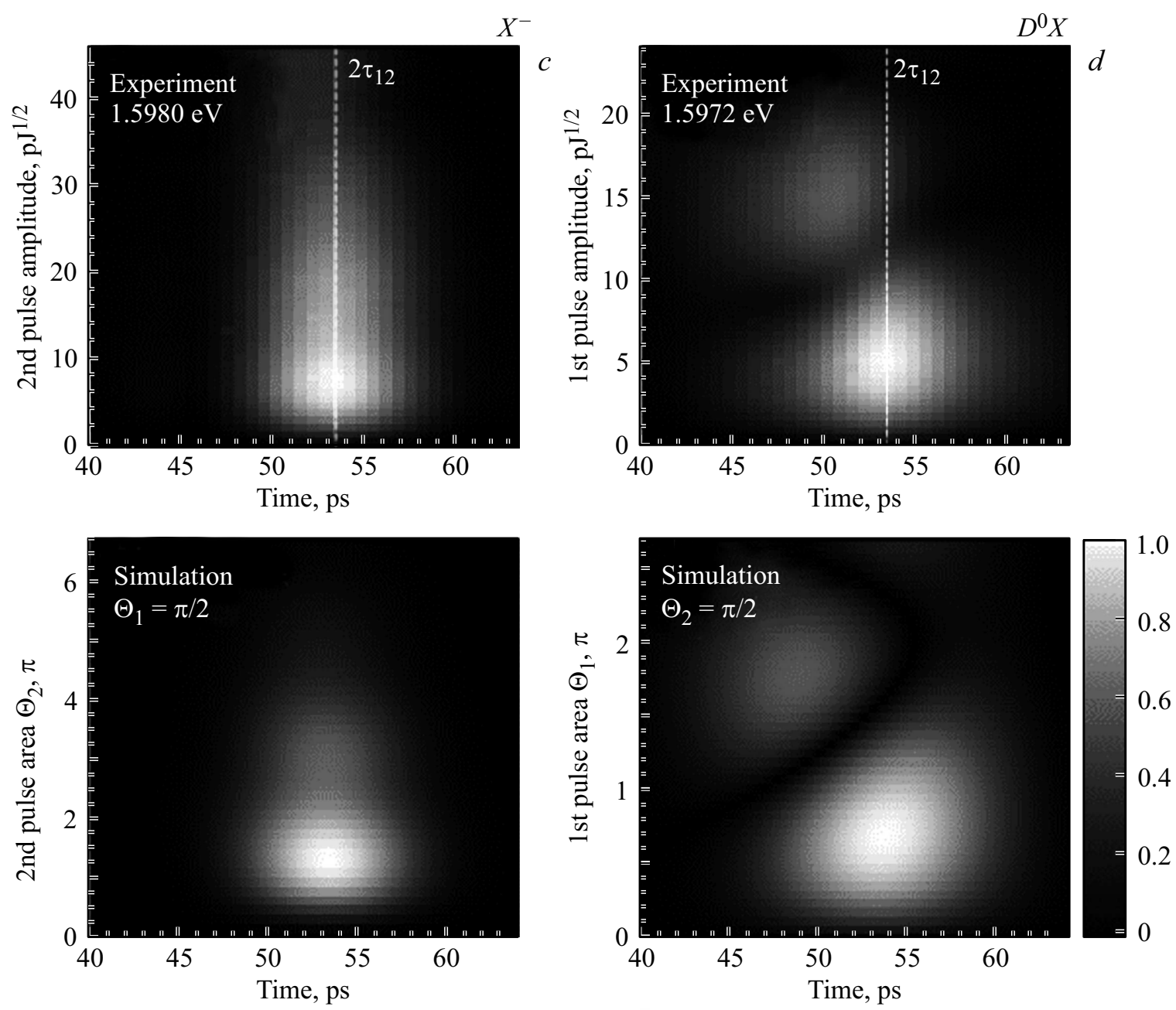

Рис. 6 (продолжение).

рис. 3, a. Возбуждение локализованного экситона с энергией $1.5985-1.5990 \mathrm{eV}$ при изменении амплитуды первого импульса не приводит к осцилляциям амплитуды эха, но приводит к быстрому затуханию эха (рис. $6, a$ ). Это обусловлено недостаточной локализацией экситонов, в результате чего возникают эффекты необратимой дефазировки, индуцированные накачкой (Excitation-Induced Dephasing, EID) [4].

При возбуждении локализованного триона ситуация радикально меняется. На рис. $6, b$ и $c$ показаны результаты измерения фотонного эха при изменении амплитуд первого и второго импульсов соответственно. В первом случае мы наблюдаем осцилляции со смещенным во времени вторым максимумом и при этом сильным затуханием амплитуды эха в силу эффектов EID. Bo втором случае временного смещения не наблюдается, а осцилляции сильно сглажены.

Временное смещение амплитуды фотонного эха при изменении амплитуды первого импульса наблюдалось нами на ансамбле квантовых точек (In, Ga) As/GaAs, помещенных в брэгговский резонатор [27]. Этот эффект обусловлен тем, что широкий спектральный ансамбль экситонов когерентно возбуждается спектрально узкими импульсами лазера. В результате уже в процессе относительно длительного возбуждения ансамбль испытывает обратимую дефазировку из-за большого разброса резонансных частот у возбуждаемых осцилляторов по сравнению с центральной частотой возбуждающего света. Когда второй возбуждающий импульс обращает временую эволюцию ансамбля, то рефазировка ансамбля происходит в момент времени, который несколько смещается относительно задержки $2 \tau_{12}$ [32]. Этот эффект, однако, удается учесть в простой модели ансамбля двухуровневых систем, когерентная динамика которых описывается оптическими уравнениями Блоха. Результат численного моделирования, описывающий экспериментальные данные для триона, показан на нижних панелях рис. $6, b$ и $c$. Помимо процессов дефазировки, индуцированной оптической накачкой, в расчетах учитывается также пространственная неоднородность пятна возбуждения, имеющего гауссово распределение интенсивности [25].

Детектирование осцилляций Раби на комплексе $D^{0} X$ качественно выглядит так же, как и на трионе, однако наблюдаются некоторые отличия. Во-первых, длительность 
импульса фотонного эха на комплексе $D^{0} X$ больше, что объясняется меньшим неоднородным уширением возбуждаемого ансамбля, чем в случае локализованных трионов. Во-вторых, дефазировка, индуцированная оптическим возбуждением, существенно меньше, в результате чего осцилляции затухают медленнее при росте амплитуды импульса.

Таким образом, результаты измерения фотонного эха показывают, что ансамбль комплексов $D^{0} X$ в квантовых ямах $\mathrm{CdTe} /(\mathrm{Cd}, \mathrm{Mg}) \mathrm{Te}$ с небольшой концентрацией доноров $\left(\sim 10^{10} \mathrm{~cm}^{-2}\right)$ является хорошей системой для получения эффективного сигнала фотонного эха при помощи последовательности двух интенсивных оптических импульсов с площадями $\pi / 2$ и $\pi$ соответственно. Однако, дальнейшее увеличение мощности возбуждения приводит к неизбежному разрушению когерентности и ослаблению сигналов фотонного эха за счет многочастичных взаимодействий и разогрева электронной системы.

Авторы работы выражают благодарность своим коллегам L. Langer, M. Salewski, T. Meier, M. Reichelt, М.М. Глазову, Л.Е. Голубу, Г.Г. Козлову и Ю.В. Капитонову.

\section{Список литературы}

[1] У.Х. Копвиллем, В.Р. Нагибаров, Физика металлов и металловедение 15, 313 (1963).

[2] N.A. Kurnit, I.D. Abella, S.R. Hartmann. Phys. Rev. Lett. 13, 567 (1964).

[3] D.A. Wiersma, K. Duppen. Science 237, 1147 (1987).

[4] D.S. Chemla, J. Shah. Nature 411, 549 (2001).

[5] V.V. Samartsev. Laser Phys. 20, 383 (2010).

[6] A.I. Lvovsky, B.C. Sanders, W. Tittel. Nature Photon. 3, 706 (2009).

[7] G. Noll, U. Siegner, S.G. Shevel, E.O. Göbel. Phys. Rev. Lett. 64, 792 (1990).

[8] D.G. Steel, S.T. Cundiff. Laser Physics 12, 1135 (2002).

[9] P. Borri, W. Langbein, S. Schneider, U. Woggon, R.L. Sellin, D. Ouyang, D. Bimberg. Phys. Rev. Lett. 87, 157401 (2001).

[10] T.H. Stievater, X. Li, D.G. Steel, D. Gammon, D.S. Katzer, D. Park, C. Piermarocchi, L.J. Sham. Phys. Rev. Lett. 87, 133603 (2001).

[11] A. Zrenner, S. Beham, E. Stufler, F. Findeis, M. Bichler, G. Abstreiter. Nature (London) 418, 612 (2002).

[12] M.O. Scully, M.S. Zubairy. Quantum Optics. Ch 7. Cambridge University Press, Cambridge, England (1997).

[13] M. Fleischhauer, A. Imamoglu, J.P. Marangos. Rev. Mod. Phys. 77, 633 (2005).

[14] M. Dyakonov. Spin Physics in Semiconductors. SpringerVerlag, Berlin (2008).

[15] A. Greilich, D.R. Yakovlev, A. Shabaev, Al.L. Efros, I.A. Yugova, R. Oulton, V. Stavarache, D. Reuter, A. Wieck, M. Bayer. Science 313, 341 (2006).

[16] S.G. Carter, Z. Chen, S.T. Cundiff. Phys. Rev. B 76, 201308 (2007).

[17] D. Press, T.D. Ladd, B. Zhang, Y. Yamamoto. Nature 456, 218 (2008).
[18] I. Broser, B. Lummer, R. Heitz, A. Hoffmann. J. Cryst. Growth 138, 809 (1994).

[19] D. Brinkmann, J. Kudrna, P. Gilliot, B. Hönerlage, A. Arnoult, J. Cibert, S. Tatarenko. Phys. Rev. B 60, 4474 (1999).

[20] G. Moody, I.A. Akimov, H. Li, R. Singh, D.R. Yakovlev, G. Karczewski, M. Wiater, T. Wojtowicz, M. Bayer, S.T. Cundiff. Phys. Rev. Lett. 112, 097401 (2014).

[21] F. Fras, Q. Mermillod, G. Nogues, C. Hoarau, C. Schneider, M. Kamp, S. Höfling, W. Langbein, J. Kasprzak. Nature Photonics 10, 155 (2016).

[22] L. Langer, S.V. Poltavtsev, I.A. Yugova, D.R. Yakovlev, G. Karczewski, T. Wojtowicz, J. Kossut, I.A. Akimov, M. Bayer. Phys. Rev. Lett. 109, 157403 (2012).

[23] L. Langer, S.V. Poltavtsev, I.A. Yugova, M. Salewski, D.R. Yakovlev, G. Karczewski, T. Wojtowicz, I.A. Akimov, M. Bayer. Nature Photonics 8, 851 (2014).

[24] M. Salewski, S.V. Poltavtsev, I.A. Yugova, G. Karczewski, M. Wiater, T. Wojtowicz, D.R. Yakovlev, I.A. Akimov, T. Meier, M. Bayer. Phys. Rev. X 7, 031030 (2017).

[25] S.V. Poltavtsev, M. Reichelt, I.A. Akimov, G. Karczewski, M. Wiater, T. Wojtowicz, D.R. Yakovlev, T. Meier, M. Bayer. Phys. Rev. B 96, 075306 (2017).

[26] S.V. Poltavtsev, A.N. Kosarev, I.A. Akimov, D.R. Yakovlev, S. Sadofev, J. Puls, S.P. Hoffmann, M. Albert, C. Meier, T. Meier, M. Bayer. Phys. Rev. B 96, 035203 (2017).

[27] S.V. Poltavtsev, M. Salewski, Yu.V. Kapitonov, I.A. Yugova, I.A. Akimov, C. Schneider, M. Kamp, S. Höfling, D.R. Yakovlev, A.V. Kavokin, M. Bayer. Phys. Rev. B 93, 121304(R) (2016).

[28] M. Salewski, S.V. Poltavtsev, Yu.V. Kapitonov, J. Vondran, D.R. Yakovlev, C. Schneider, M. Kamp, S. Höfling, R. Oulton, I.A. Akimov, A.V. Kavokin, M. Bayer. Phys. Rev. B 95, 035312 (2017).

[29] A.A. Sirenko, T. Ruf, M. Cardona, D.R. Yakovlev, W. Ossau, A. Waag, G. Landwehr. Phys. Rev. B 56, 2114 (1997).

[30] E.A. Zhukov, D.R. Yakovlev, M. Bayer, M.M. Glazov, E.L. Ivchenko, G. Karczewski, T. Wojtowicz, J. Kossut. Phys. Rev. B 76, 205310 (2007).

[31] J.M. Kikkawa, D.D. Awschalom. Phys. Rev. Lett. 80, 4313 (1998).

[32] В.В. Самарцев, Р.Г. Усманов, Г.М. Ершов, В.Ш. Хамидуллин. ЖЭТФ 74, 6, 1979 (1978).

Редактор Е.Ю. Флегонтова 